\title{
Study of Atherogenic Index of Plasma (AIP) and Other Lipid Indices in Helicobacter Pylori Infection
}

\author{
Dr. Arpitha MR ${ }^{1}$, Dr. Shubha Jayaram ${ }^{2 *}$, Dr. Meera $S^{3}$, Dr. Sreenivas $N^{4}$, Dr. Lokesh M. G ${ }^{5}$, Dr. Deepa K ${ }^{6}$ \\ ${ }^{1}$ Postgraduate student, ${ }^{2}$ Professor, Department of Biochemistry, ${ }^{3}$ Professor \& Head, Department of Biochemistry, ${ }^{4}$ Professor, Department of Pathology, \\ ${ }^{5}$ Assistant Professor, Department of Surgery, ${ }^{6}$ Assistant Professor, Department of Biochemistry, Mysore Medical College \& Research Institute, Mysore, \\ Karnataka -570001
}

\author{
DOI: $10.36348 /$ sijb.2019.v02i12.007 \\ *Corresponding author: Dr. Shubha Jayaram
}

| Received: 20.12.2019 | Accepted: 27.12.2019| Published: 30.12 .2019

\section{Abstract}

Helicobacter pylori (H. pylori) is a gram negative bacterium that naturally colonizes the gastric epithelium, which causes chronic gastritis and peptic ulcer disease. Recent studies have shown that it may interfere with many biological processes and influence the occurrence of many diseases outside the stomach. Many studies have proposed a link between $H$. pylori infection and atherosclerosis. Atherogenic Index of Plasma (AIP) has been considered as a strong marker to predict the risk of atherosclerosis. The present study was done to assess the correlation between AIP and other lipid indices with $H$. pylori infection. The study comprised of 50 biopsy proven $H$. pylori cases and 50 age-sex matched controls. Blood samples were collected in fasting state and analyzed for total cholesterol (TC), triglycerides (TG), HDL-cholesterol (HDL) and LDL-cholesterol and the lipid indices were calculated. Lipid indices like AIP, CRI 1 \& 2 \& AC were significantly higher in $H$. pylori infected cases compared to controls. Hence these lipid indices can be used for identifying individuals at higher risk of cardiovascular diseases in $H$. pylori infected patients.

Keywords: Helicobacter pylori, Atherosclerosis, Atherogenic index of Plasma (AIP), Castelli Risk Index (CRI), Atherogenic Coefficient(AC), lipid indices, Coronary Artery Disease(CAD).

\begin{abstract}
Copyright @ 2019: This is an open-access article distributed under the terms of the Creative Commons Attribution license which permits unrestricted use, distribution, and reproduction in any medium for non-commercial use (NonCommercial, or CC-BY-NC) provided the original author and source are credited.
\end{abstract}

\section{INTRODUCTION}

Helicobacter pylori (H. pylori) is a gramnegative bacterium that is widespread all over the world, infecting more than $50 \%$ of the world's population [1]. This causes chronic gastritis and peptic ulcer disease and this organism has also been implicated as a risk factor for developing gastric cancer, which is the second most frequent cause of cancerrelated death [2]. H. pylori infection affects gastric physiology [3] and also associated with lipid metabolism, elevated fasting insulin levels and insulin sensitivity [1].

The effect of $H$. pylori infection on the serum lipid profile is still a matter of debate. Several studies have demonstrated that $H$. pylori infection might modify serum lipid concentrations. Blood cholesterol levels, such as elevated low density lipoprotein (LDL) and decreased high density lipoprotein (HDL) are major risk factors for cardiovascular diseases [4]. Despite such studies, in the absence of an abnormal lipid profile the possibility of CAD cannot be ruled out. It has been suggested that the different combinations of these lipid profile parameters can be used to identify such high risk individuals [5]. Atherogenic Index of Plasma (AIP), Castelli Risk Index 1\&2 (CRI 1\&2) and Atherogenic Coefficient (AC) are the ratios that have been studied as markers of lipid atherogenic risk. These are the calculated fractions which can be used in the clinical setting for assessing the risk of cardiovascular disease beyond the routinely done lipid profile.

Atherogenic Index of Plasma (AIP) is based on two important parameters TG and HDLc, both of which are independent risk factors for CAD. Castelli Risk Index (CRI-I) calculated as (TC/HDL) and CRI-II as (LDL/HDL), which involves independent risk factors for $\mathrm{CAD}$. Atherogenic Coefficient (AC) calculated as $\{(\mathrm{TC}-\mathrm{HDL}) / \mathrm{HDL}\}$ is another ratio relying on the significance of HDL in predicting the risk of CAD [6].

So the present study has been undertaken to analyse the various lipid indices like Atherogenic Index of Plasma (AIP), Castelli Risk Index 1 \& 2 (CRI 1 \& 2) $\&$ Atherogenic Coeficient (AC) in assessing the risk of CAD in H. pylori. 


\section{OBJECTIVES}

- To calculate the lipid indices like Atherogenic Index of plasma (AIP), Castelli's Risk Index (CRI) I, Castelli's Risk Index (CRI) II \& Atherogenic Coeficient (AC) in H. pylori infected individuals.

- Comparing these indices with age and sex matched controls

\section{MATERIALS AND METHODS}

The study was conducted in the Department of Biochemistry, MMC\&RI Mysore. This case-control study was undertaken after obtaining the Institutional ethical clearance. The study was conducted from February 2019 to July 2019.

Sample size: The sample size was estimated using estimation technique with an absolute error $15 \%$, $5 \%$ alpha error. The sample size was estimated using the following formula,

$$
\mathrm{n}=\mathrm{z}^{2} \frac{p q}{d^{2}}
$$

Where,

$\mathrm{n}=$ Sample size

$\mathrm{z}=$ Standard Normal Deviate $[\mathrm{z}$ value $]$ for $\mathrm{a}$ given level of confidence

$$
\begin{aligned}
& \mathrm{p}=\text { prevalence or proportion } \\
& \mathrm{q}=1-\mathrm{p} \\
& \mathrm{d}=\text { absolute allowable error }
\end{aligned}
$$

A total of 125 subjects were enrolled in our study after informed written consent. We have taken endoscopic gastric biopsy from patients with chronic gastritis and biopsy samples were subjected to histopathological examination. Based on the histopathological findings the study subjects were divided into two groups, 50 biopsy proven $H$. pylori positive cases and 50 age- sex matched $H$. pylori negative controls.

\section{Exclusion Criteria}

- Patients on hypolipidemic drugs.

- Patients with chronic diseases like Diabetes mellitus, chronic kidney disease and liver disease.

- Known cases of cardiovascular diseases and cerebrovascular diseases.

- Pregnant and lactating females.

Collection of Blood Sample: $3 \mathrm{ml}$ of blood was collected under aseptic precautions. Serum was separated after centrifugation and analysed. The serum total cholesterol was estimated by enzymatic method, serum triglycerides by enzymatic GPO-PAP method, serum HDL cholesterol by Direct enzymatic method and LDL cholesterol was calculated by FredricksonFriedwald formula \{total cholesterol - HDL cholesterol - (triglycerides/5)\}. Then Lipid indices were calculated by following formulas.

Atherogenic Index of Plasma $=\log (\mathrm{TG} / \mathrm{HDL})[6]$

Castelli Risk Index I = TC / HDL [6]

Castelli Risk Index II = LDL / HDL [6]

Atherogenic Coefficient $=(\mathrm{TC}-\mathrm{HDL}) / \mathrm{HDL}[6]$

Statistical Method: Statistical analysis was performed with the GraphPad Instat version 3.1 software programme. For comparison of variables with a normal distribution unpaired 2-tailed Student's t-test was used, whereas the Mann-Whitney U-test was used for variables with a skewed distribution. A $p \leq 0.05$ was considered statistically significant.

\section{RESULTS}

Table $1 \&$ fig. 1 shows gender distribution and mean age in controls and cases. Out of 50 controls $54 \%$ were males, $46 \%$ were females \& out of 50 cases $52 \%$ were males, $48 \%$ were females.

Table-1: Comparison of gender distribution and mean age in controls and case

\begin{tabular}{|l|l|l|l|l|}
\hline & \multicolumn{2}{|l|}{$\begin{array}{l}\text { Controls (50) } \\
\text { H. pylori negative }\end{array}$} & \multicolumn{2}{l|}{$\begin{array}{l}\text { Cases (50) } \\
\text { H. pylori positive }\end{array}$} \\
\hline & Males & Females & Males & Females \\
\hline No. & 27 & 23 & 26 & 24 \\
\hline$\%$ & 54 & 46 & 52 & 48 \\
\hline Age & $48.62 \pm 14.13$ & $46.17 \pm 12.8$ & $50.34 \pm 12.12$ & $44.08 \pm 11.41$ \\
\cline { 2 - 3 }
\end{tabular}

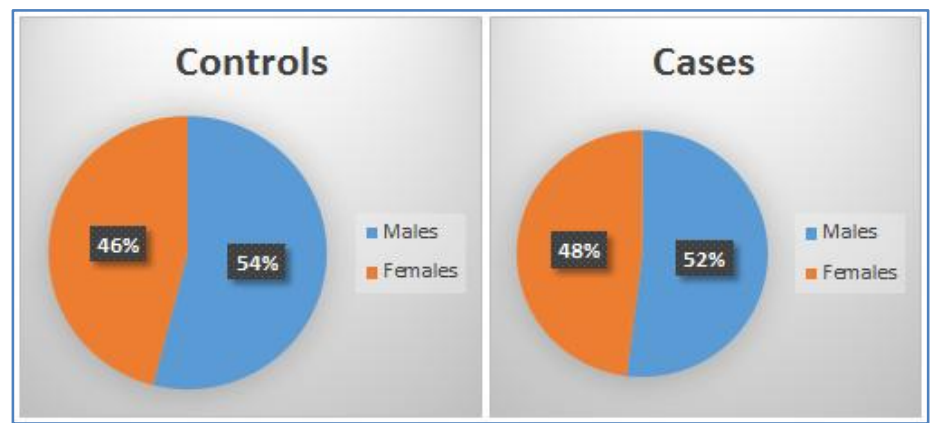

Fig-1: Showing males to female ratios in controls and case 
Table 2, fig $2 \& 3$ shows Lipid indices in controls and cases, H. pylori positive group had statistically significant increase in AIP, CRI 1, CRI 2 \& AC when compare to controls.

Table-2: Comparison of lipid indices in controls and case

\begin{tabular}{|l|l|l|}
\hline & Controls & Cases \\
\hline AIP (Atherogenic Index of plasma) & $0.106 \pm 0.22$ & $0.246 \pm 0.219^{*}$ \\
\hline CRI I (Castelli's Risk Index I) & $3.852 \pm 1.244$ & $4.806 \pm 1.348^{*}$ \\
\hline CRI II (Castelli's Risk Index II) & $2.212 \pm 1.00$ & $2.862 \pm 1.348^{*}$ \\
\hline AC (Atherogenic Coeficient) & $2.946 \pm 1.276$ & $3.978 \pm 1.846^{* *}$ \\
\hline
\end{tabular}

*significant; **highly significant

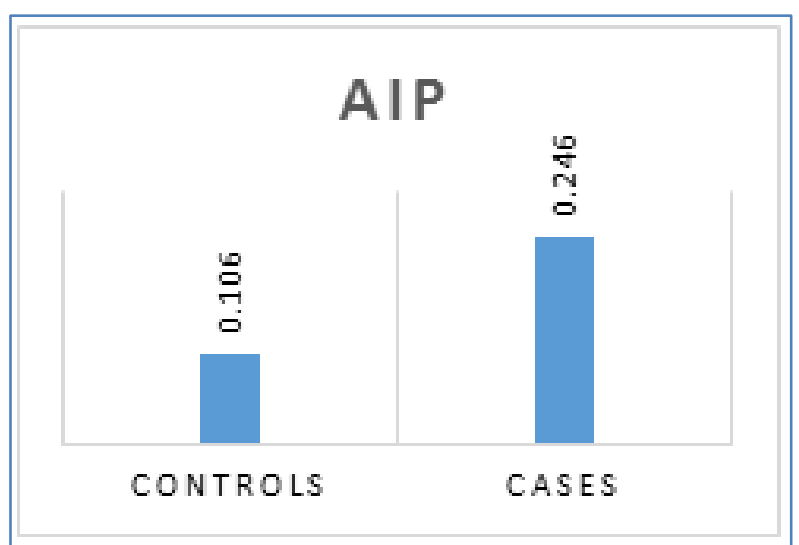

Fig-2: Comparison of AIP values in controls and case

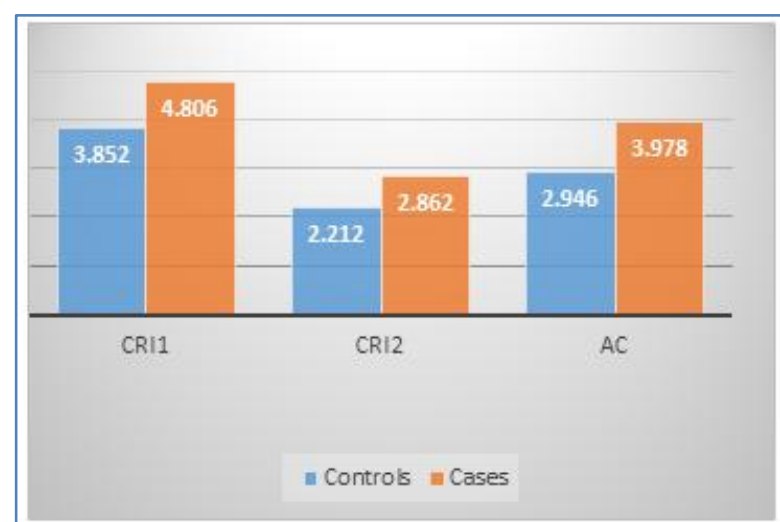

Fig-3: Comparison of CRI I \& II, AC values in controls and case

Table 3 \& fig4 depicts risk stratification of cases based on AIP levels. Among cases 13 were at low risk, 15 intermediate risk and 22 were at high risk.

Table-3: Risk stratification of cases based on AIP levels [7]

\begin{tabular}{|l|l|}
\hline AIP & Cases \\
\hline$<0.1$ & $13(26 \%)$ \\
Low risk & \\
\hline $0.1-0.24$ & $15(30 \%)$ \\
Intermediate risk & \\
\hline$>0.24$ & $22(44 \%)$ \\
High risk & \\
\hline
\end{tabular}

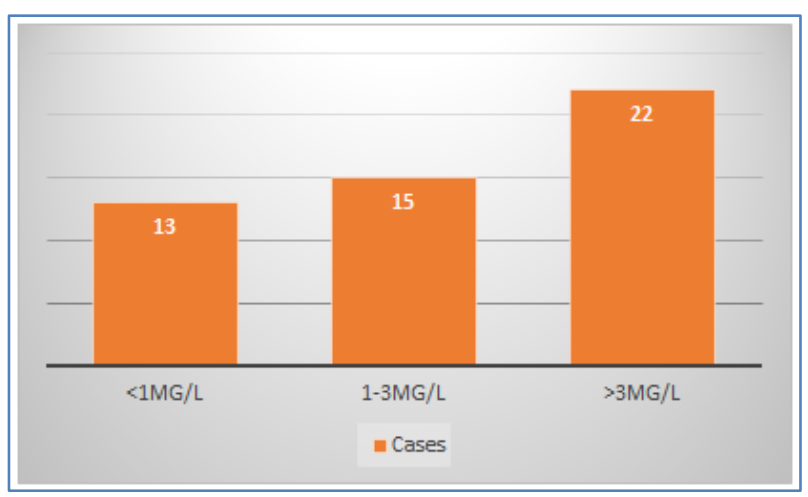

Fig-4: Risk stratification of cases based on AIP levels

\section{DISCUSSION}

Many studies have proposed a link between $H$. pylori infection and risk of atherosclerosis \& coronary heart diseases. The results of Danesh et al. on the relation of cardiovascular disease and $H$. pylori showed a significant increase and direct relation of cardiovascular disease and $H$. pylori infection [8]. To understand the relation between $H$. pylori and risk of coronary artery diseases we tested serum lipid levels and calculated the lipid indices in $H$. pylori positive \& negative subjects. Results showed there was statistically significant relationship between lipid indices in the patients (case) groups as compared with the control group.

The exact pathophysiology underlying the change in the level of serum lipids was not fully understood. Different mechanisms were suggested, including imbalance between synthesis and utilization of plasma lipids, usage of lipids to restore damaged cell membranes, and interaction of cytokines and bacterial toxins with lipids [9]. Alterations in the composition and function of lipoproteins, due to decreased reverse cholesterol transport and increased oxidation of lipids occurs by bacterial infection [10].

Table $1 \&$ figure 1 shows that among cases $52 \%$ were males and $28 \%$ were females and the mean age group of cases was 47 years and majority (56\%) were aged 31-50years. The present study result is concordant with a study done by Siddiqui B et al. [11].

Cholesterol/HDL ratio and LDL/HDL ratio are risk factors for developing cardiovascular diseases [12]. The Canadian working group had chosen the TC/HDL 
ratio as a secondary goal of therapy considering it to be a more sensitive and specific index of cardiovascular risk than TC [13]. Concerning these ratios, our study showed a statistically significant difference (CRI 1 $3.852 \pm 1.244$ in controls \& $4.806 \pm 1.348$ in cases \& CRI2 $2.212 \pm 1.00$ in controls \& $2.862 \pm 1.348$ in cases). Hoffmeister et al. [14] and Niemel et al. [15] also showed similar results. Ansari M et al. [12] showed increase in Cholesterol/HDL ratio and LDL/HDL ratio in cases compared to controls. Adachi $\mathrm{K}$ et al. [16] concluded that the LDL/HDL ratio in the H. pylori-positive group was significantly higher than that in the $H$. pylori-negative group, and successful $H$. pylori eradication tended to reduce that ratio.

Many studies have shown that AIP is an indirect indicator of small dense LDL levels. The value of AIP indicates a balance between the actual concentration of plasma TG and HDL, which predetermine the direction of the cholesterol transport in the intravascular pool (i.e the flux of newly produced cholesteryl esters by lecithin cholesterol acyltransferase) toward atherogenic LDL or beneficial HDL[17]. AIP is being used by some practitioners as a significant predictor of atherosclerosis. It has been suggested that AIP values of $<0.1$ are associated with low, 0.1 to 0.24 with medium and above 0.24 with high cardiovascular risk[7]. We have studied AIP \& observed a significant increase in an AIP ratio of 0.246 in cases when compared to controls (0.106).

Atherogenic Coefficient (AC), calculated as $\{(\mathrm{TC}-\mathrm{HDL}) / \mathrm{HDL}\}$ is a measure of cholesterol in LDL, VLDL, IDL lipoprotein fractions with respect to HDL. It reflects atherogenic potential of the entire spectrum of lipoprotein fractions [5]. In the present study, AC was significantly elevated in cases compared to controls. As the ratio is based on Non-HDL, the statistically significant increase of $\mathrm{AC}$ as seen in the study further strengthens the atherogenic risks in $H$. pylori.

\section{CONCLUSION}

The above observations suggest that lipid ratios like Atherogenic Index of Plasma, Castelli risk index I \& II and Atherogenic coefficient can be used for identifying individuals at higher risk of cardiovascular diseases in $H$. pylori infected patients. Early detection $\&$ treatment of high risk individuals can help in the reduction in the morbidity, mortality and good prognostic outcome.

\section{ACKNOWLEDGEMENT}

Authors would like to thank Dr. Dinesh H. N., Professor \& Head, Dept of Surgery, MMC\&RI, Mysore and all the patients participated in this study.

\section{REFERENCES}

1. Franceschi, F. (2014). Role of Helicobacter pylori infection on nutrition and metabolism. World Journal of Gastroenterology, 20(36), 12809.

2. Essawi, T., Sarari, A., Farraj, M., \& Hamoudi, W. (2008). Helicobacter pylori, a causative agent of vitamin B12 deficiency. The Journal of Infection in Developing Countries, 2(05).

3. Annibale, B., Capurso, G., Martino, G., Grossi, C., \& Delle Fave, G. (2000). Iron deficiency anaemia and Helicobacter pylori infection. International Journal of Antimicrobial Agents, 16(4), 515-519.

4. Cullen, P., \& Assmann, G. (1999). High risk strategies for atherosclerosis. Clinica Chimica Acta, 286(1-2), 31-45.

5. Bhardwaj, S., Bhattacharjee, J., Bhatnagar, M., \& Tyagi, S. (2013). Atherogenic Index of Plasma, Castelli Risk Index and Atherogenic CoefficientNew Parameters in Assessing Cardiovascular Risk. International Journal of Pharmacy and Biological Sciences, 3(3), 359-364.

6. Patil, M., Jayaram, S., Meera S., \& Kantharaj N. (2015). Role of novel Lipid indices and Lipoprotein (a) in type -II Diabetes Mellitus with coronary artery disease. Indian Journal of Fundamental And Applied Life Sciences, 5(2), 4147.

7. Sein, M., Ohnmar, \& Latt, T. (2015). Association of waist circumference with atherogenic cardiovascular risks in centrally obese Myanmar male subjects. International Journal of Clinical and Experimental Physiology, 2(1), 46-50.

8. Danesh, J., Collins, R., \& Peto, R. (2000). Lipoprotein (a) and Coronary Heart Disease. Circulation, 102(10), 1082-1085.

9. Nassaji, M., \& Ghorbani, R. (2012). Plasma lipid levels in patients with acute bacterial infections. Turk J Med Sci, 42(3), 465-469.

10. Khovidhunkit, W., Memon, R., Feingold, K., \& Grunfeld, C. (2000). Infection and Inflammation-Induced Proatherogenic Changes of Lipoproteins. The Journal of Infectious Diseases, 181(3), 462-S472.

11. Siddiqui, B., Yakoob, J., Abbas, Z., Azmat, R., Fatima, S., \& Awan, S. (2018). Distribution of Helicobacter pylori infection and abnormal bodymass index (BMI) in a developing country. The Journal of Infection in Developing Countries, 12(05), 342-346.

12. Ansari, M., Omrani, M., Sayyah, B., \& Ansari, S. (2010). Effect of Helicobacter pylori infection on the lipid, lipoproteins, apolipoprotein-A1, Lipoprotein (a) and Apolipoprotein-B in patients with gastritis. African Journal of Microbiology Research, 4(1), 84-87.

13. Genest, J., Frohlich, J., Fodor, G., \& McPherson, R. (2003). Recommendations for the management of dyslipidemia and the prevention of cardiovascular disease: summary of the 2003 update. CMAJ, 169(9), 921-924. 
14. Hoffmeister, A., Rothenbacher, D., Bode, G., Persson, K., März, W., \& Nauck, M. (2001). Current Infection WithHelicobacter pylori, but Not Seropositivity toChlamydia pneumoniaeor Cytomegalovirus, Is Associated with an Atherogenic, Modified Lipid Profile. Arteriosclerosis, Thrombosis, And Vascular Biology, 21(3), 427-432.

15. Niemela, S., Karttunen, T., Korhonen, T., Laara, E., Karttunen, R., Ikaheimo, M., \& Kesaniemi, Y. (1996). Could Helicobacter pylori infection increase the risk of coronary heart disease by modifying

serum

lipid concentrations?. Heart, 75(6), 573-575.

16. Adachi, K., Mishiro, T., Toda, T., Kano, N., Fujihara, H., \& Mishima, Y. (2018). Effects of Helicobacter pylori eradication on serum lipid levels. Journal of Clinical Biochemistry and Nutrition, 62(3), 264-269.

17. Mannangi, N., Jayaram, S., HS, V., Kashinakunti, S., \& R, M. (2015). Novel Lipid Indices in Chronic Kidney Disease. National Journal of Medical Research, 5(1), 39-42. 